\title{
Facilitated Gesture Recognition Based Interfaces for People with Upper Extremity Physical Impairments
}

\author{
Hairong Jiang ${ }^{1}$, Juan P. Wachs ${ }^{1}$, and Bradley S. Duerstock ${ }^{1,2}$ \\ ${ }^{1}$ School of Industrial Engineering, Purdue University, West Lafayette, IN, USA \\ ${ }^{2}$ Weldon School of Biomedical Engineering, Purdue University, West Lafayette, IN, USA \\ \{jiang115, jpwachs, bsd \} @purdue. edu
}

\begin{abstract}
A gesture recognition based interface was developed to facilitate people with upper extremity physical impairments as an alternative way to perform laboratory experiments that require 'physical' manipulation of components. A color, depth and spatial information based particle filter framework was constructed with unique descriptive features for face and hands representation. The same feature encoding policy was subsequently used to detect, track and recognize users' hands. Motion models were created employing dynamic time warping (DTW) method for better observation encoding. Finally, the hand trajectories were classified into different classes (commands) by applying the CONDENSATION method and, in turn, an interface was designed for robot control, with a recognition accuracy of $97.5 \%$. To assess the gesture recognition and control policies, a validation experiment consisting in controlling a mobile service robot and a robotic arm in a laboratory environment was conducted.
\end{abstract}

Keywords: Gesture recognition, particle filter, dynamic time warping (DTW), CONDENSATION.

\section{Introduction}

Effective, natural and intuitive human computer interfaces (HCI) are critical aspects in the development of assistive technologies [1]. Voice, facial expressions, gaze and hand gestures have been widely used as communication channels for unimodal or multimodal interfaces for people with upper mobility impairments. Those interfaces were used for intelligent wheelchairs control, wellness monitoring and home medical alert systems [2,3], to mention a few. Upper limb gesturing is of particular use, since people already use gestures for communicating desired actions (e.g. pointing destination, indicating direction and motion), thus having to learn a series of atypical body movements is avoided. For those individuals who are able to move their hands and upper arms to some degree, gesture-based HCI is an extremely promising alternative or complement to existing interface techniques.

Hand gesture recognition algorithms involve the hand segmentation, tracking, and trajectories recognition. A common method for hand segmentation involves modeling the user skin color [4]. Depending purely on color information is unreliable, brightness, unstructured backgrounds, and clutter affects object segmentation. If the focus is on the gestures' trajectories, instead of the hand shape, classic tracking approaches 
can be adopted. For example, Camshift is a well-established and basic algorithm for object tracking and was previously used for hand tracking [5]. Other more complex and robust approaches include the CONDENSATION algorithm developed by Isard and Black [6]. Particle filter is a common stochastic based technique for object tracking that can be easily parallelized. Perez applied the color-based appearance model to a particle filter framework to enhance tracking under complex backgrounds and occlusions [7]. In terms of gestures classification, the predominant approach is still Hidden Markov Models (HMM) (see Bilal for an extensive review of HMM applied to hand posture and gesture recognition [8]). Common problems with HMM involve finding a good set of parameters (e. g. initial probabilities) and trajectory spotting for gesture temporal segmentation. Black and Jepson [9] proposed a CONDENSATIONbased trajectory gesture recognition algorithm to this end. Nevertheless the gestures segmentation was not addressed in that work.

In this paper, particle filters and the CONDENSATION algorithm were combined for hand tracking and gesture classification in a simplistic yet robust fashion, which made it appropriate for human robot interaction (HRI) in assistive technologies.

\section{System Architecture}

The architecture of this system is illustrated in Fig. 1. The hand gesture based recognition system includes foreground segmentation, color-based detection, tracking, and trajectories recognition. A detailed description of the system is given in Section 3 .

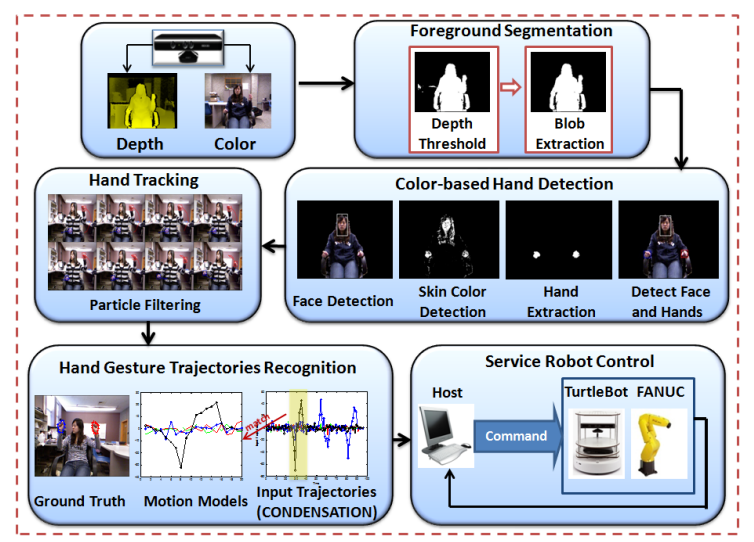

Fig. 1. System Overview

\section{Gesture Recognition}

\subsection{Foreground Segmentation}

To detect the user's movements, the user's body was treated as the foreground object. Two steps were employed to segment the foreground (refer to algorithm 1). The first step was to exclude pixels based on their distance to the camera (depth thresholding). 
The second step required ruling out small areas and keeping the largest blob in the remaining image as the foreground (blob cleaning). In the first step, the depth information was assessed through a Kinect sensor (fig. 2(a)). Two absolute depth thresholds (a low threshold $\mathrm{T}_{\mathrm{DL}}$ and a high threshold $\mathrm{T}_{\mathrm{DH}}$ ) were manually set by the user according to their relative distance to the sensor. $\mathrm{T}_{\mathrm{DL}}$ is set to no less than a constant which is the minimum distance that can be observed by the depth sensor (due to its physical limitations). $\mathrm{T}_{\mathrm{DH}}$ is set to be the maximum distance that can be reached by the user ${ }^{1}$. Only those pixels with a depth value between the two thresholds were kept in a mask image (fig. 2(b)). The mask image was used to compute the area of the biggest region (blob), denoted as $\left(\mathrm{B}_{\mathrm{SH}}\right)$. All the remaining blobs with a smaller area than $\mathrm{B}_{\mathrm{SH}}$ were deleted (fig. 2(c)). If the largest blob is not the face or hands, it will be discarded in a later stage since tracking is performed based on color and spatial information.

\section{Algorithm 1: Foreground Segmentation}

Input: $\mathrm{T}_{\mathrm{DL}} ; \mathrm{T}_{\mathrm{DH}}$; depth Image $\mathrm{D}(\mathrm{i}, \mathrm{j})$;

$\mathrm{D}_{1}(\mathrm{i}, \mathrm{j})=\left\{\begin{array}{lr}1: & T_{D L} \leq D(i, j) \leq T_{D H} \\ 0: & \text { otherwise }\end{array}\right.$

$\mathrm{T}_{\mathrm{SH}}=\max \left(\operatorname{Area}\left(\mathrm{B}_{\mathrm{i}}\right)\right) \quad / / \mathrm{B}_{\mathrm{i}}$ is the $\mathrm{i}^{\text {th }}$ blob in the mask image $\mathrm{D}_{1}$

$D_{2}(i, j)=\left\{\begin{array}{rc}1: & D_{1}(i, j) \in B_{i} \& \text { Area }\left(B_{i}\right)==T_{B H} \\ 0: & \text { otherwise }\end{array}\right.$

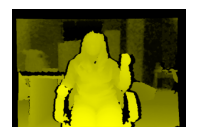

(a)Depth Image

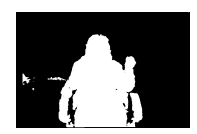

(b) Depth Threshold Mask

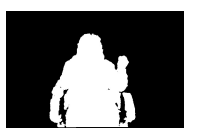

(c) Foreground Mask

Fig. 2. Foreground Segmentation

\subsection{Hand Detection}

Before detecting the hands, a face detection method [10] was used to obtain the initial face region (as shown in fig. 3(a)). The result was used to remove the face region from the target image. Skin and non-skin color histogram models were constructed by using the Compaq database [11]. The probability of a pixel to be part of the hand was calculated as the division of the two histograms (which is a proxy of the distinctiveness- the higher the ratio, the more likely the two pixels belong to different color distributions). The mask image was obtained by applying the histogram ratio and back-projecting the probabilities of each pixel back in the image (as show in fig. $3(\mathrm{~b})$ ). To obtain the hand regions without the face, the region detected by the face detector was removed from the target image. After this, the two largest blobs were selected as hand regions (fig. 3(c), (d)). This hand detection procedure is only used to provide automatic initialization to particle filter tracking. Afterwards the hands positions were assessed through continuous tracking done by the particle filter.

\footnotetext{
${ }^{1}$ These values were chosen since they resulted in the best performance; however other thresholds will impact the results differently.
} 


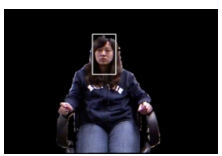

(a)Face Detection

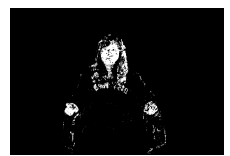

(b) Skin Color Detection

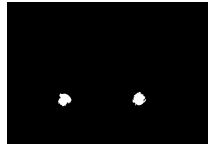

(c) Hand Extraction

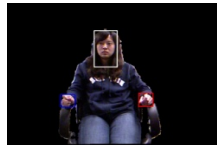

(d) Localization

Fig. 3. Face and Hand Detection

\subsection{Hand Tracking}

A color and spatial information based Sequential Importance Resampling (SIR) particle filter framework was proposed to track both face and hands through frames in video sequences $[7,12]$. The Particle filter algorithm consists of three main phases: predicting, measuring and resampling. In the prediction phase, a second order autoregressive (AR) was selected as the dynamic motion model as in equation (1):

$$
x_{t+1}=A_{1}\left(x_{t}-x_{0}\right)+A_{2}\left(x_{t-1}-x_{0}\right)+x_{0}+B v_{t}, v_{t} \sim \mathcal{N}(0, \Sigma)
$$

where $\mathrm{A}_{1}, \mathrm{~A}_{2}, \mathrm{~B}$ and $\Sigma$ were the parameter matrices that best matched the real motion of the tracked object; $\mathrm{x}$ was the state of particles. In the measuring stage, both color and spatial information were incorporated in the particle filter framework to calculate the likelihood function. The method in [8] was used to calculate the color likelihood function:

$$
\omega^{i} \propto \exp \left(-\lambda \mathrm{D}_{\mathrm{i}}^{2}\right)
$$

where $\lambda$ is the Bhattacharyya similarity coefficient ((is empirically set to 20) following the practice in [8] which delivered satisfactory results). The spatial likelihood function included three parts: the Euclidian distance between the centroid of face and hand blob (defined as $\mathrm{D}_{\mathrm{fhx}}, \mathrm{x}=1$ or 2), the two hands blobs (defined as $\mathrm{D}_{\mathrm{hh}}$ ) and each particle and the centroid of blob in the previous video frame (defined as $\mathrm{D}_{\mathrm{pc}}$ ). The spatial part of likelihood is then:

$$
\omega^{i} \propto \exp \left(\mathrm{k}_{1} \mathrm{D}_{\text {fhx }}+\mathrm{k}_{2} \mathrm{D}_{\mathrm{hh}}+\mathrm{k}_{3}\left(1 / \mathrm{D}_{\mathrm{pc}}\right)\right)
$$

Combining equation (2) and (3), the likelihood function is:

$$
\omega^{i}=\beta * \exp \left(-\lambda \mathrm{D}_{\mathrm{i}}^{2}+\mathrm{k}_{1} \mathrm{D}_{\mathrm{fhx}}+\mathrm{k}_{2} \mathrm{D}_{\mathrm{hh}}+\mathrm{k}_{3}\left(1 / \mathrm{D}_{\mathrm{pc}}\right)\right)
$$

where $\beta$ is a normalization factor; $\mathrm{k}_{1}, \mathrm{k}_{2}, \mathrm{k}_{3}$ are parameters that were set to change the weight of each feature for optimal tracking.

\subsection{Hand Trajectory Classification}

The positions of the hands in each frame of the video sequence were acquired from the tracking stage. The motion model for each gesture trajectory was created based on the data collected from eight subjects. Although gestures performed by each subject may have similar trajectories, the precise duration of each sub-trajectory within the trajectory were different. To normalize the trajectories, temporal alignment was conducted. The dynamic time warping (DTW) method was employed to accommodate differences in timing between different trajectories to the construct motion models [13]. The following procedure was proposed to obtain the motion models (Algorithm 2). 


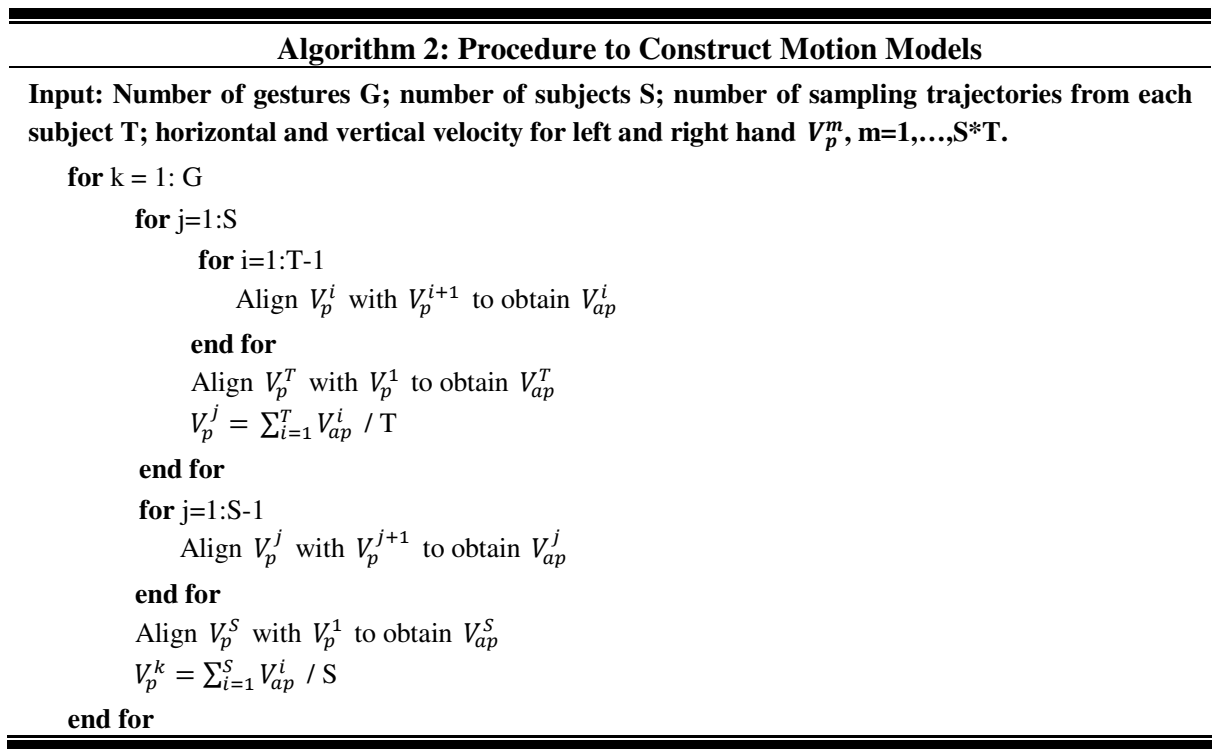

The CONDENSATION algorithm [9] was used to recognize the hand gesture trajectories. The original algorithm was extended to work for two hands. A state at time $t$ is described as a parameter vector:

$$
\mathrm{s}_{\mathrm{t}}=\left(\mu, \Phi^{x}, \alpha^{x}, \rho^{x}\right)
$$

Where, $\mu$ was the index of the motion models, $\phi$ was the current phase in the model, $\alpha$ was an amplitude scaling factor, $\rho$ was a time dimension scaling factor, $\mathrm{x}$ denoted the hand used $\mathrm{x} \in$ \{left hand, right hand\}.

\section{Experimental Results}

\subsection{Recognition Accuracy}

An eight-gesture lexicon (as shown in fig. 4) was selected according to results from an interview with individuals with physical disabilities. The size of the gesture lexicon is eight because more gestures were difficult for the users to remember, while less was not enough due to the number of functions required. It was tested by eight users and resulted in an average cross validation accuracy of 97.5\%. Ten sessions were used for cross validation of each gesture ( $\mathrm{k}$-fold with $\mathrm{k}=10$ ). In each session, 72 gestures were used for training and 8 gestures were used for testing. A confusion matrix was computed using a temporal window size of $\mathrm{w}=20$ (fig. 5). The ROC curve to demonstrate the system performance was obtained by changing the size of the window from 10 to 24 to different values (fig. 6). 


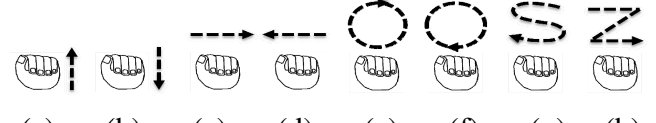
(a)
(b)
(c)
(d)
(e) (f)
(g) (h)

Fig. 4. Gesture Lexicon

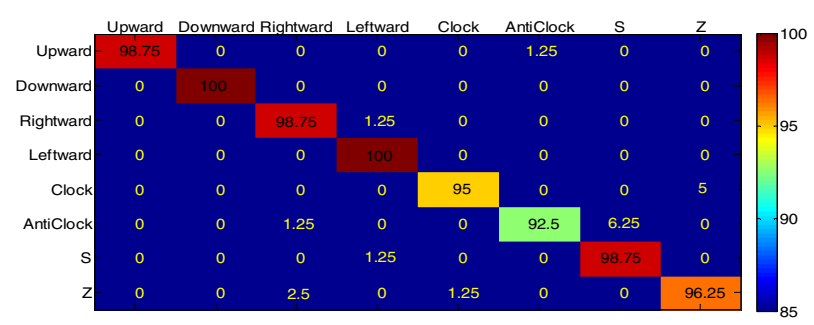

Fig. 5. Confusion Matrix with the window size- $w=20$

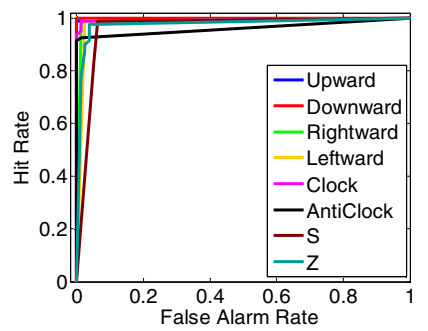

Fig. 6. ROC Curve showing recognition performance for each gesture

\subsection{Laboratory Experiment}

A laboratory case study was completed five times by one able bodied individual with each mode of operation. While the task completion time depends on the user performing the experiment, general trends can be observed in this pilot experiment regarding task completion time that can be generalized to larger pools of users. In this experiment, a mobile robot was controlled by the gesture algorithm to transport a beaker to a position near a robotic arm. The robotic arm would then be activated by the operator to add a reagent to the beaker and the mobile robot would be summoned back to the beginning point. To control the mobile robot and the robotic arm, the gestures in the lexicon from (a)-(h) were mapped to the following commands: 'change mode', 'robotic arm action', 'go forward', 'go backward', 'turn left', 'turn right', 'stop' and 'enable robotic arm'. Two modes were used to control the mobile robot: discrete and continuous mode. In discrete mode, the robot moved an increment of distance, every time that a command was issued. While the continuous mode, the robot responded to the given command, until the 'stop' command was issued. To switch between these two modes one distinctive gesture ('upward') was used. In the experiment, the discrete, continuous and combined (continuous plus discrete) control modes were tested. The resulting average task completion times were of 205, 109.8 and 143.2 seconds, 
respectively (fig. 7). Each recognition process required only $47 \mathrm{~ms}$ for face and both hand recognition. The map for the lab and the trajectories of the robot for discrete (red star line), continuous (blue solid line), and combined (black dash line) control modes were recorded to test for correlation between average completion time for a task with fixed distance and the used control mode (fig. 8).

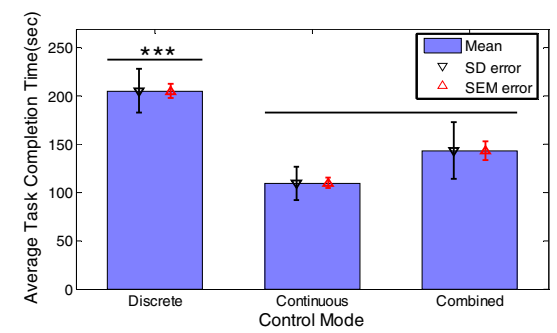

Fig. 7. Average Task Completion Time, Unpaired t-test, $\mathrm{p}<0.001$

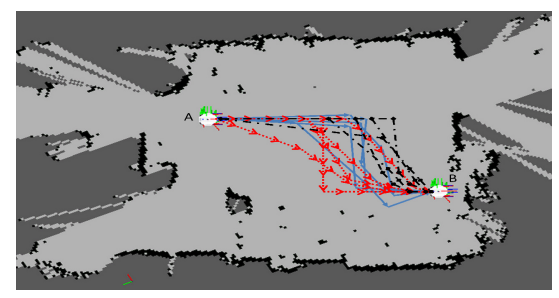

Fig. 8. Robot trajectories for different control modes

\section{$5 \quad$ Conclusions and Future Work}

A hand gesture recognition based interfaces was developed for people with limited upper limb mobility. The depth information was used to segment the human body from a non-static background. An automatic initialization procedure for the particle filter method was engineered by combining blob extraction, face detection, image dilation, erosion and color histograms techniques. Both color and spatial information were considered when applying the particle filter framework. A training procedure was proposed to obtain motion models for each gesture in the lexicon. The CONDENSATION algorithm was used to classify the bimanual gestures. The gesture recognition algorithm designed was found to reach a recognition accuracy of $97.5 \%$. A laboratory task experiment was conducted to validate real time performance of the gesture interface to assist in conducting a typical biomedical lab procedure with the help of two robots. From the results, the continuous mode required the least average task completion time, while the discrete control mode required the most. Therefore, the authors recommend continuous control mode is used most of the time and the discrete is used only when the robot is very near to the target. Future work includes: (1) Determining automatically the depth thresholds for the Kinect sensor and their effect in the segmentation results. (2) Study additional robust techniques for hand 
tracking to tackle the problem of resilience to occlusions (when one hand occludes the other). (3) Recruit more users in the future versions of system for gesture lexicon testing to enhance the system's robustness.

Acknowledgement. This work was partially funded by the National Institutes of Health NIH Director's Pathfinder Award to Promote Diversity in the Scientific Workforce, grant number DP4-GM096842-01.

\section{References}

1. Jacko, J.A.: Human-Computer Interaction Design and Development Approaches. In: 14th HCI International Conference, pp. 169-180 (2011)

2. Moon, I., Lee, M., Ryu, J., Mun, M.: Intelligent Robotic Wheelchair with EMG-, Gesture-, and Voice-based Interfaces. In: International Conference on Intelligent Robots and Systems, pp. 3453-3458. IEEE Press (2003)

3. Reale, M., Liu, P.: Yin. L.J.: Using eye gaze, head pose and facial expression for personalized non-player character interaction. In: IEEE Computer Society Conference on Computer Vision and Pattern Recognition Workshops, pp. 13-18. IEEE Press (2011)

4. Soriano, M., Martinkauppi, B., Huovinen, S., Laaksonen, M.: Skin detection in video under changing illumination conditions. In: 15th International Conference on Pattern Recognition, vol. 1, pp. 839-842 (2000)

5. Bradski, G.R.: Computer vision face tracking as a component of a perceptual user interface. In: Workshop on Applications of Computer Vision, Princeton, NJ, pp. 214-219 (1998)

6. Isard, M., Black, A.: CONDENSATION: Conditional density propagation for visual tracking. International Journal of Computer Vision 29, 5-28 (1998)

7. Pérez, P., Hue, C., Vermaak, J., Gangnet, M.: Color-Based Probabilistic Tracking. In: Heyden, A., Sparr, G., Nielsen, M., Johansen, P. (eds.) ECCV 2002, Part I. LNCS, vol. 2350, pp. 661-675. Springer, Heidelberg (2002)

8. Bilal, S., Akmeliawati, R., Shafie, A.A., Salami, M.J.E.: Hidden Markov Model for human to computer interaction: a study on human hand gesture recognition. In: Artificial Intelligence (2011)

9. Black, M.J., Jepson, A.D.: A Probabilistic Framework for Matching Temporal Trajectories: CONDENSATION-Based Recognition of Gestures and Expressions. In: Burkhardt, H.-J., Neumann, B. (eds.) ECCV 1998, Part I. LNCS, vol. 1406, pp. 909-924. Springer, Heidelberg (1998)

10. Viola, P., Jones, M.: Rapid object detection using a boosted cascade of simple features. In: International Conference on Computer Vision and Pattern Recognition, pp. 511-518 (2001)

11. Jones, M.J., Rehg, J.M.: Statistical color models with application to skin detection. In: IEEE Computer Society Conference on Computer Vision and Pattern Recognition, vol. 46, pp. 81-96 (2002)

12. Hess, R., Fern, A.: Discriminatively Trained Particle Filters for Complex Multi-Object Tracking. In: IEEE Computer Society Conference on Computer Vision and Pattern Recognition, pp. 240-247 (2009)

13. Aach, J., Church, G.M.: Alignment gene expression time series with time warping algorithms. J. Bioinformatics 17(6), 495-508 (2001) 